G. Zhang, T. Yang, S. Gregori, J. Liu, F. Maloberti: "Ultra-low power motion triggered image sensor for distributed wireless sensor network"; Proc. of IEEE Sensors 2003, Toronto, 22-24 October 2003, Vol. 2, pp. 1141-1146.

(C)20xx IEEE. Personal use of this material is permitted. However, permission to reprint/republish this material for advertising or promotional purposes or for creating new collective works for resale or redistribution to servers or lists, or to reuse any copyrighted component of this work in other works must be obtained from the IEEE. 


\title{
ULTRA-LOW POWER MOTION-TRIGGERED IMAGE SENSOR FOR DISTRIBUTED WIRELESS SENSOR NETWORK
}

\author{
Guangbin Zhang, Tianhong Yang, Stefano Gregori, Jin Liu, Franco Maloberti \\ Electrical Engineering Department, University of Texas at Dallas, Richardson, Texas, USA \\ gbzhang, thy021000, Stefano, jinliu, franco.maloberti@utdallas.edu
}

\begin{abstract}
This paper describes a motion-triggered CMOS image sensor. It uses low resolution photosensor array with an ultra low power data converter in the read out circuit. The ana$\log$ motion sensor shares the same photosensors using an interleaved structure. The motion sensor triggers the digital imager and RF data transmission to the base station in case there are objects moving in the scene, otherwise, the system remains standby to save power. This design is specially optimized for very low power applications such as distributed wireless sensor network.
\end{abstract}

\section{Keywords}

Low power, CMOS imager, motion sensor

\section{INTRODUCTION}

This paper describes the integrated electronic circuits of a generic sensor platform for a distributed wireless sensor network. The device is powered by on-board microbatteries which are remotely rechargeable via microwave from the base station. Since the radiation regulation limits the maximum microwave power, the available power for the remote sensors is extremely low. In this paper, we focus on an ultra-low power motion triggered image sensor.

Fig. 1 presents the sensor module architecture. The power is provided by microwaves from the base station while a RF signal is used to send data to the base station. A DSP will be used to control the functioning of the whole sensor and the communication with the base station. A power management unit manages the power supply of the chips. The module is intended for any possible plug-in sensor. However, optical sensors are the most challenging elements in terms of power consumption and required transmission bandwidth. The total power budget is $100 \mu \mathrm{W}$. By optimizing the pixel and A/D converter design, we obtained a very low power digital 2-D image sensor which dissipates less than $35 \mu \mathrm{W}$. However, when the imaging acquisition and transmission is on, the DSP and RF part consume much more power, exceeding $100 \mu \mathrm{W}$ when certain data rate is required. Fortunately, for a security camera surveillance system, it is not necessary to keep the camera and RF data transmission on all the time, unless there are movements in the scene. So, when the scene is stationary, it is possible we turn off the digital camera system and go to standby mode. When the system is in acquisition mode, it is easy for the base station to send a command to the re- mote sensor to enter standby mode when it finds that the scene is quiet. A low power motion sensor could act as a monitor and wake up other parts of the device when there are movements. We designed an analog motion sensor which shares the photo sensors with the digital imager using an interleaved structure. Since most of the time the scene is static the system can remain standby to save large portion of power. The designed motion sensor dissipates only $20 \mu \mathrm{W}$, which allows the device to transfer up to 80 percent of the microwave power to micro-battery for later usage. Besides lowering down the power consumption, it also reduces the amount of information to be transmitted, thus, reduces the total bandwidth requirement for RF communication.

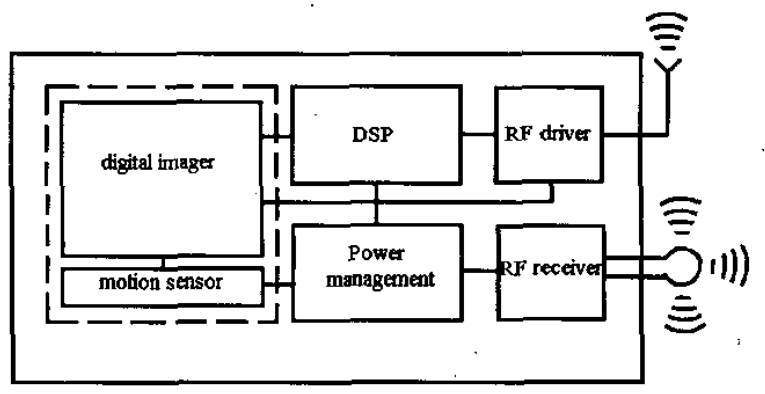

Fig. 1. Motion triggered image sensor for distributed wireless sensor network

\section{ARCHITECTURE OF MOTION TRIGGERED IMAGE SENSOR}

Fig. 2 shows the interleaved structure for the motion sensing operation. An additional transistor is added into each pixel to set the operation modes. In acquisition mode, we sample each pixel frame by frame using a column/row selection circuit and a single ADC to obtain digitized data. In standby mode, the digital camera is off, and the added transistors are turned on to connect the photo current outputs to the motion sensor cells. We connect every other pixel in the even columns to the $\mathrm{x}$-axis 1-D motion sensor array to detect horizontal motion, and connect every other pixel in the even row to the $y$-axis 1-D motion sensor array to detect vertical motion, respectively. A decision circuit compares the motion sensor output with a threshold to decide whether to turn on the system or not. Since the digital imager and the motion sensor are integrated on a single chip and share the same set of photosensors, only one set of 
optical lenses is required to realize compact design. The picture shows only a $6 \times 6$ array as an example, but the actual demo image sensor contains a digital imager of $64 \mathrm{x}$ 64 photodiodes and two $16 \times 1$ analog motion sensor array, with layout on a $2 \mathrm{~mm}$ by $2 \mathrm{~mm}$ mini-chip in TSMC $0.18 \mu \mathrm{m}$ process.

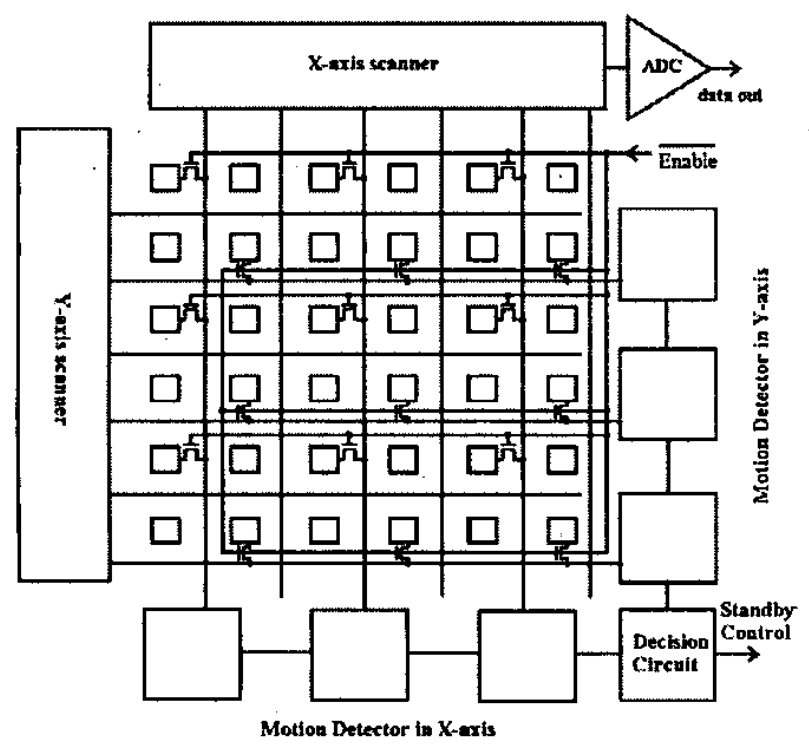

Fig. 2. Interleaved structure of combined digital imager and analog motion sensor

\section{THE LOW POWER DIGITAL IMAGER}

Fig. 3 shows typical structure of a passive pixel image sensor. The pixel array shares one charge amplifier which consume the largest portion of power in this architecture. To minimize the power, the proposed schematic is shown in Fig. 4. The entire array shares one single A/D converter and the charge amplifier is eliminated.

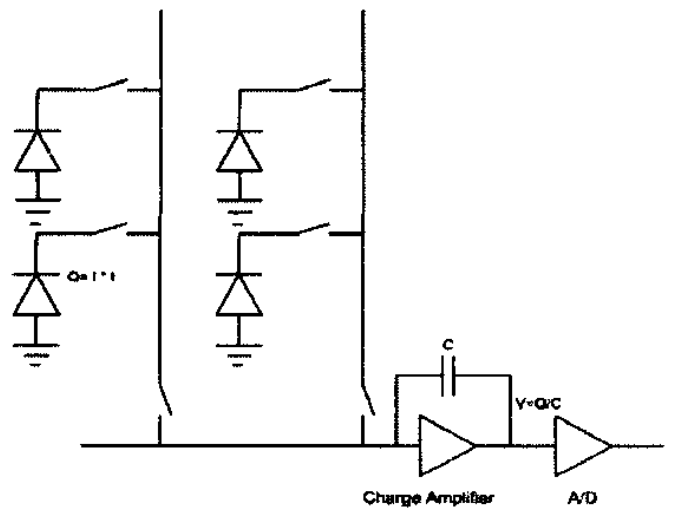

Fig.3. Passive pixel image sensor structure

Pixel design and readout circuit

Different from conventional solutions, this design uses a successive approximation $\mathrm{A} / \mathrm{D}$ converter. As mentioned before, the system measures the change resulting from the integration of the photo current in a storing capacitor $\mathrm{C}_{\mathrm{s}}$ directly and does not require a charge amplifier. When a given pixel is selected, the charge on the storing capacitor is shared with the capacitors array of the successive approximation network. Since the storing capacitor equals the value of the entire SAR array, the signal is divided by two. In reality, if accounting for the parasitic effects, the final read voltage, $V_{X}$, is about half of the original voltage, $\mathrm{V}$, stored in $\mathrm{C}_{\mathrm{s}}$. This relationship can be expressed as

$$
\mathrm{V}_{\mathrm{x}}=\mathrm{v} \frac{C_{s}}{2 C_{s}+C_{p}},
$$

where $\mathrm{C}_{\mathrm{s}}$ is $0.4 \mathrm{pF}$ and $\mathrm{C}_{\mathrm{p}}$ is the sum of the diode capacitance and 64 transistor diffusion-substrate capacitances. In this case, $\mathrm{C}_{\mathrm{p}}$ is around $170 \mathrm{fF}$, and the final read out voltage is 0.4 times of the original voltage stored in $\mathrm{C}_{\mathrm{s}}$. To make the ratio close to 0.5 , larger $C_{s}$ can be used.

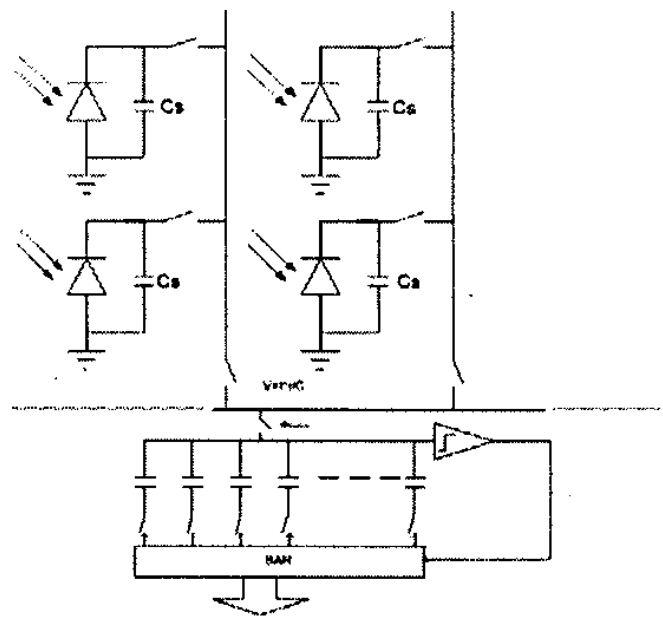

Fig.4. Proposed sensor structure

The layout area of each pixel is $12 \times 12 \mu \mathrm{m}^{2}$. The photodiode are Nwell-Psub, single ring structure. This structure is chosen due to its better responsivity (measured from previously fabricated chip) and good isolation between the pixels [1]. Since the average photo current is around $0.7 \mathrm{nA}$, the calculated power consumption of photodiodes of $64 \mathrm{x}$ 64 array is $3.44 \mu \mathrm{W}$.

\section{Low power ADC}

A significant fraction of the power consumption of image sensor array comes from the analog to digital interface. This chip as already mentioned does not require a charge pre-amplifier, while using a low power comparator in a conventional successive approximation (SA) converter. Fig. 5 shows the SA architecture. The comparator has zero DC current and uses capacitors instead of active loads. A charging/discharging process enables amplifying the input signal. Simulation shows it has 8-bit resolution, averaged 
$5.7 \mu \mathrm{W}$ analog and $4.0 \mu \mathrm{W}$ digital power consumption at $200 \mathrm{kHz}$ conversion frequency.

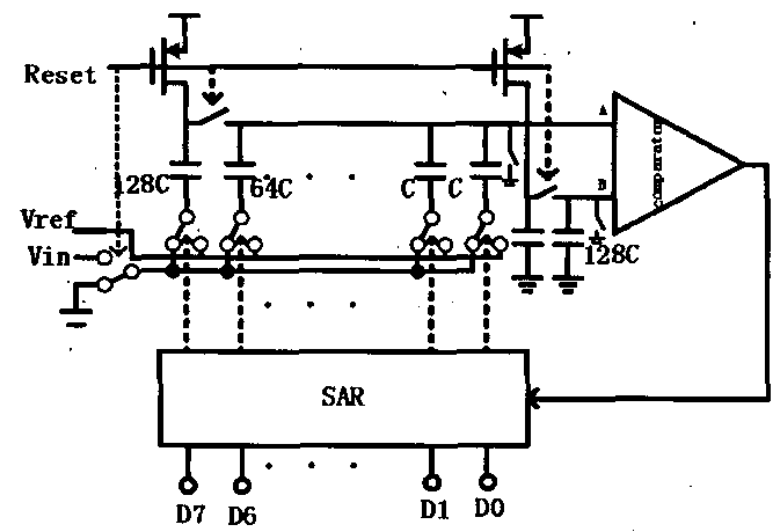

Fig.5. Low power charge redistribution successive approximation converter

The schematic of comparator used into the $\mathrm{ADC}$ is shown in Fig 6. During $\Phi 1$, the two switches across $C_{1}$ and $C_{2}$ discharge the capacitors and bring the differential output to $\mathrm{V}_{\mathrm{DD}}$. The transistors controlled by $\Phi 2$ keep the input pair off. During $\Phi 2$, the voltage $\mathrm{Vb}$ enables M3 and M4 to form cascode configurations with $\mathrm{M} 1$ and M2. Capacitors $C_{1}$ and $C_{2}$ are charged and the output voltages go down. M5 and M6 make a common mode feedback and control the quiescent current across the differential input. At a given point, the current goes to zero and the output voltages reach a steady value. A possible unbalancement of the input voltages leads to an amplified steady output difference. The outputs are then used to drive a latch controlled by the phase $\Phi_{\mathrm{L}}$. The power consumption of the preamplifier is $P_{p}=C_{1} V_{D D}{ }^{2} f$. In our design $\mathrm{C}_{1}=\mathrm{C}_{2}=200 \mathrm{fF}$, $\mathrm{V}_{\mathrm{DD}}=1.5 \mathrm{~V}, \mathrm{f}=2 \mathrm{MHz}$. therefore, $\mathrm{P}_{\mathrm{p}}=0.9 \mu \mathrm{W}$. The power required by the positive-feedback latch is $1.1 \mu \mathrm{W}$.

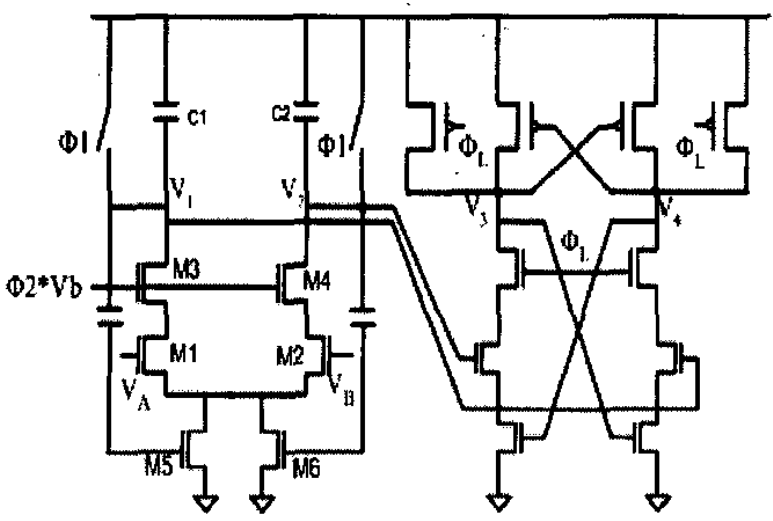

Fig.6. Low power comparator

The control logic is generated through successive approximate register (SAR [3]) by registering the comparator out- put every bit cycle. Then, the binary weighted capacitor changed the $V_{A}$ of the comparator.

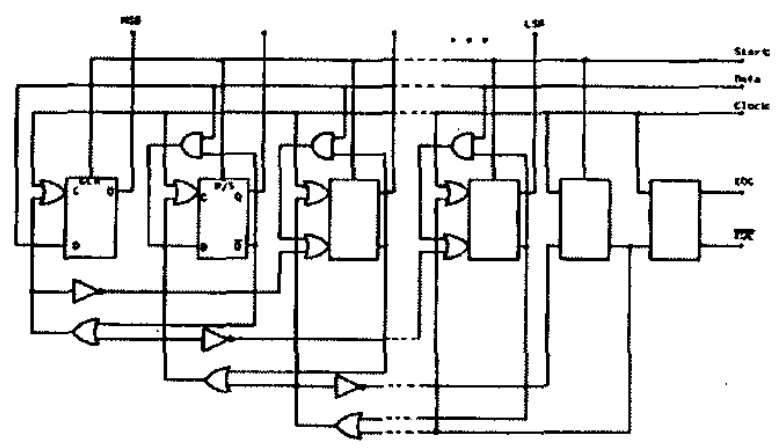

Fig.7. Structure of SAR

Fig. 8 shows some simulated waveforms for the ADC. The difference between input signal and the common voltage ( $\mathrm{Vdd} / 2$ in this architecture) is amplified about ten times by the preamplifier. (a) shows the preamplifier outputs. They dynamically change and they are suitable for controlling a positive feedback latch. (b) shows the latch outputs which are triggered by $\Phi_{\mathrm{L}}$. (c) shows the two inputs signal of the comparator in Fig 5. " $B$ " terminal is kept constant and " $A$ " terminal is changed by SAR according to the comparator output bit. Then the correct output bit is obtained from the SAR through an output buffer.

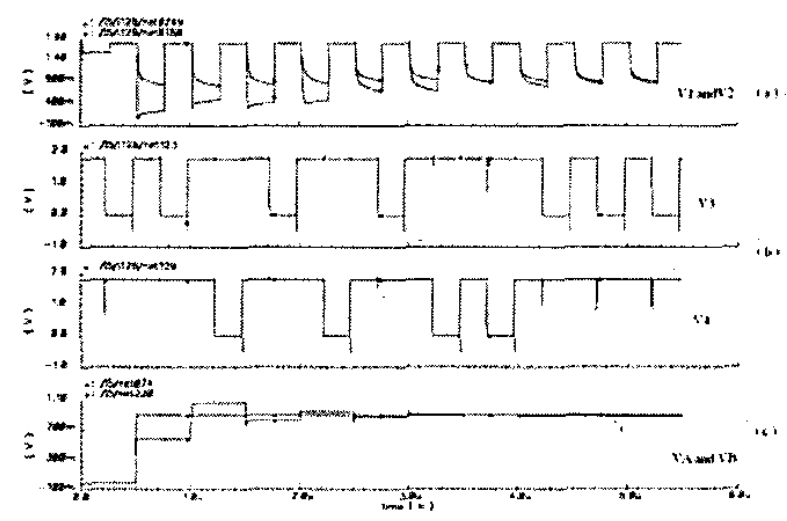

Fig.8. Simulation results for the ADC (a) output signals of preamplifier (b) output signals of latch (c) input signals of the comparator

Fig. 9 shows the response time of the positive-feedback latch stage. It takes about $4 \mathrm{~ns}$ to establish the logic signal with input difference $50 \mathrm{mV}$. However, when the difference of two inputs is $1 \mathrm{LSB}$, i.e. $4 \mathrm{mV}$, the time will increase to $5 \mathrm{~ns}$. And the reason that the latch stage in Fig. 6 is chosen is that the outputs of the pre-amplifier are always high in half time and gradually goes low in another half [4][5]. This schematic ensures that there is no static power consumption when the latch works in hold mode. When the latch is triggered, the logic level is achieved in 4ns, so it 
only consumes power during the short switching time. An additional SR latch follows the latch shown in Fig. 6.

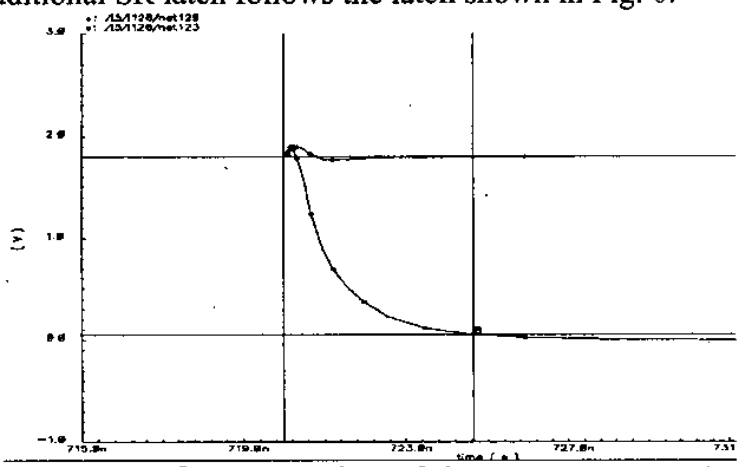

Fig.9. Response time of the comparator

Table 1 summarizes the simulated ADC performances. The total power consumption of the 2-D image sensor comes from the pixels $(3.5 \mu \mathrm{W})$, the $\mathrm{ADC}(9.5 \mu \mathrm{W})$, the switching matrix $(10 \mu \mathrm{W})$ and the data decoder $(10 \mu \mathrm{W})$. Therefore, the estimated power consumption is $33 \mu \mathrm{W}$.

Table 1. Specifications of the low power ADC

\begin{tabular}{|c|c|}
\hline Sampling frequency & $200 \mathrm{kHz}$ \\
\hline Resolution & 8 bit \\
\hline Process & $0.18 \mu \mathrm{m}$ CMOS \\
\hline Power supply & $1.5 \mathrm{~V}$ \\
\hline Power consumption & $\begin{array}{c}5.672 \mu \mathrm{W} \text { analog } 4.007 \mu \mathrm{W} \\
\text { digital }\end{array}$ \\
\hline Figure of Merit & $0.189 \mathrm{pJ} /$ conversion \\
\hline
\end{tabular}

THE LOW POWER ANALOG MOTION SENSOR CMOS motion sensor chips have been developed during the past 15 years [6][7][8][9][10]. Many visual chips detect motion by tracking the disappearance of an edge and its subsequent reappearing at neighboring pixel, using an edge detector. Typical design of the edge detector compares the concurrent signal with the temporally delayed version, or spatially smoothed version to find out edges. However, these approaches normally lead to active delay elements or filters, which require some of the transistors working in saturation region. A typical value of the power consumption per pixel of these motion sensors is about $50 \mu \mathrm{W}$, thus, the power consumption for a $32 \times 1$ motion sensor array is about $1600 \mu \mathrm{W}$. This is acceptable for most applications, but not for the RF powered distributed wireless sensor system which requires extreme low power operation. One solution to reduce the power is to use fewer pixels. However, very low resolution may decrease the spatial sensitivity, since the small area movements may not necessarily cause obvious change of luminance for large area. In addition, when the sensor resolution is too low, it cannot distinguish whether a large area light density change is caused by the change of the environment luminance such as adjusting the brightness of a lamp or by motion.

To overcome these difficulties while keep the power consumption very low, a new motion detection method is proposed by detecting a moving edge. A static edge in the image is defined sufficient light density contrast between neighboring pixels; the moving of the edge is detected based on the change of the contrast between neighboring pixels. In this approach, the moving edge is detected by directly comparing the outputs of the neighboring pixels without using active delay elements or filter circuits to generate a second version of signal to compare with. As a result, this design largely reduces the power consumption by using simple static logic circuit.

Fig. 10 shows the architecture of the 1-D motion sensor. The edge detectors use the outputs from neighbor pixels to detect the moving edge. The detected moving edge pulses from different locations are then added up together by a pulse fusing circuit. The output of the fusing circuit drives the decision circuit to count the pulses by charging a capacitor. Then, the voltage on this capacitor is compared with a threshold voltage pre-set by the user. Once it reaches a certain voltage level, it means there are substantial movements in the scene so the motion sensor will output an 'enable' signal to trigger the digital image acquisition to start. A $1.5 \mathrm{~V}$ power supply is used for all parts of the motion sensor.

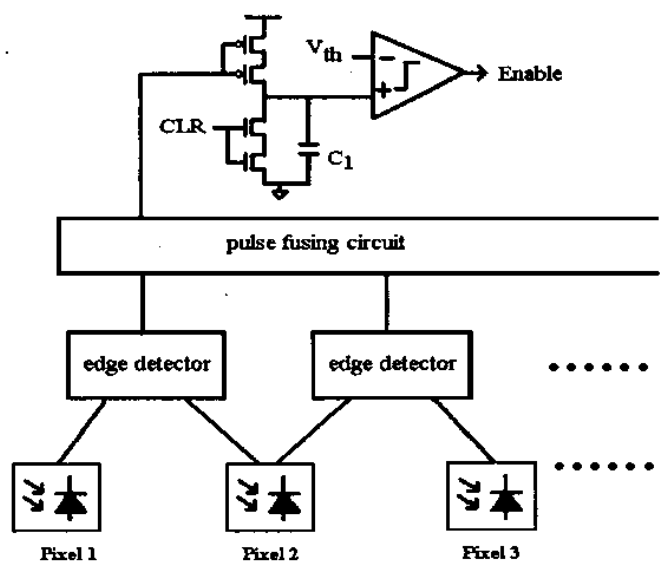

Fig. 10. Motion detection and trigger circuit.

\section{Motion edge detector}

Fig. 11 shows the schematic of the edge detector. The photocurrent is applied to two cascaded diode-mode P-type MOSFETs to obtain log scale current-voltage mapping. Simulation shows it has 6 decades or $120 \mathrm{~dB}$ of linear re- 
sponse range (from $1 \mathrm{pA}$ to $1 \mu \mathrm{A}$ ) with $205 \mathrm{mV}$ voltage drop per decade. A Hysteresis comparator is used to compare the neighboring pixel outputs. The Hysteresis shift voltage is $\mathrm{V}_{\mathrm{sh}}= \pm 10 \mathrm{mV}$.

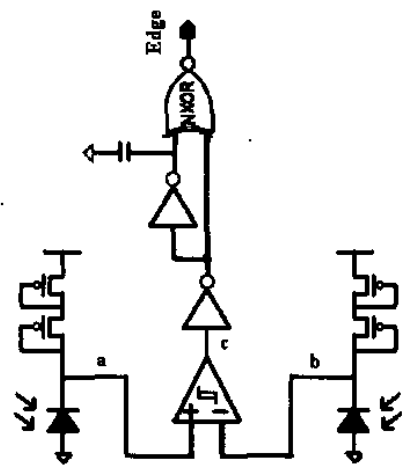

Fig. 11. Edge detector

If the output of the neighboring pixels as marked as ' $a$ ' and ' $b$ ', and the output of the comparator is marked as 'c', the motion detection algorithm is shown as Table 2 .

Table 2. Motion detection algorithm

\begin{tabular}{|l|l|l|l|l|}
\hline $\begin{array}{l}\text { Previous } \\
\text { inputs }\end{array}$ & $\begin{array}{l}\text { Previous } \\
\text { output }\end{array}$ & $\begin{array}{l}\text { Current } \\
\text { inputs }\end{array}$ & $\begin{array}{l}\text { Current } \\
\text { output }\end{array}$ & $\begin{array}{l}\text { Motion } \\
\text { edge }\end{array}$ \\
\hline $\mathrm{a} \leq \mathrm{b}+\mathrm{V}_{\mathrm{sh}}$ & $\mathrm{C}=$ low & $\mathrm{a}>\mathrm{b}+\mathrm{V}_{\text {sh }}$ & $\mathrm{c}=$ high & Yes \\
\hline $\mathrm{a} \geq \mathrm{b}+\mathrm{V}_{\mathrm{sh}}$ & $\mathrm{C}=$ high & $\mathrm{a}<\mathrm{b}+\mathrm{V}_{\text {sh }}$ & $\mathrm{c}=$ low & Yes \\
\hline $\mathrm{a} \leq \mathrm{b}+\mathrm{V}_{\mathrm{sh}}$ & $\mathrm{C}=$ low & $\mathrm{a} \leq \mathrm{b}+\mathrm{V}_{\text {sh }}$ & $\mathrm{c}=$ low & No \\
\hline $\mathrm{a} \geq \mathrm{b}+\mathrm{V}_{\mathrm{sh}}$ & $\mathrm{c}=$ high & $\mathrm{a} \geq \mathrm{b}+\mathrm{V}_{\text {sh }}$ & $\mathrm{c}=$ high & No \\
\hline
\end{tabular}

In this design with $205 \mathrm{mv} /$ decade current/voltage mapping and $\pm 10 \mathrm{mv}$ hysteresis, the theoretical sensible contrast change for the detection of moving edges is $12 \%$. However, the mismatch of the fabrication may cause the actual sensible contrast to increase. For example, assume that the maximum mismatch causes $10 \mathrm{mv}$ offset in pixel output, the worst case sensible contrast then increases to $25 \%$. But since the mismatches are normally randomly distributed, some of the pixel sensitivities will drop while some others may even increase. Motion with contrast change lower than the hysteresis plus mismatch will not be detected. Looking into the algorithm in Table 2, we can also see that there will be possible edge missing in some conditions. For example, when ' $a$ ' is already larger than ' $b$ ' $+V_{\text {sh }}$, and later on ' $a$ ' becomes even larger because of object moving, this motion will not be detected by the circuit because it causes no transient change at the comparator output. However, this is tolerable since the motion sensor has two 1-D arrays, and the motion is very likely to be caught at other points with different backgrounds. More complex algorithm could be developed to reduce the edge missing rate, however, tradeoffs with power consumption needs to be considered.
After the comparator, an NXOR based digital edge detector is used to convert each rising and falling edges of the comparator output to a single narrow pulse. The pulse width is tuned by adjusting the output impedance of the inverter and the load capacitor; in this design, it is $20 \mathrm{~ns}$.

\section{Pulse fusion circuit and decision circuit}

Fig. 12 shows the schematic of the pulse fusion and decision circuit. Most of the time the N-MOSFETs $m_{1} \sim m_{k}$ controlled by the detected edge pulses are turned off so that the voltage on $\mathrm{C}_{3}$ remains high. When a moving edge occurs, one of the N-MOSFETs $m_{1} \sim m_{k}$ is turned on to discharge $C_{3} . C_{3}$ will be recharged to high voltage when all $m_{1}$ to $m_{k}$ are off. Since all the drain nodes of $m_{1}$ to $m_{k}$ are connected together to $C_{3}$, the pulses are fused into one pulse. However, since the charging process of $\mathrm{C}_{3}$ is much slower than the discharging process, the pulse width on $\mathrm{C}_{3}$ will be expanded compared with the original pulses. Then, a second digital pulse extractor is used to obtain a fixed width narrow pulse. This pulse extractor is similar to the one used previously, but, a NAND gate is used instead of NXOR gate for extracting the falling edges only. The obtained short pulse is fixed at $17 \mathrm{~ns}$ pulse width. We use this pulse to control the step-by-step charging process of $C_{1} \cdot C_{1}$ can also be discharged by 'Clear' signal from the DSP to reinitiate the motion detection process. The PMOSFET and NMOSFET connected to $C_{1}$ are in cascode structure in order to reduce the leakage current when both the P-type and N-type MOSFETs are off and the charge on $\mathrm{C}_{1}$ will be maintained for some time. The decision whether to wake up the digital imager is based on the comparison of the voltage on $\mathrm{C}_{1}$ with a threshold voltage. The threshold voltage, Vth, is adjustable by user externally. It can be configured to ignore certain level of non-continuous or small scale motion by setting $V$ th higher than quiescent voltage across $C_{1}$ when no edges occur.

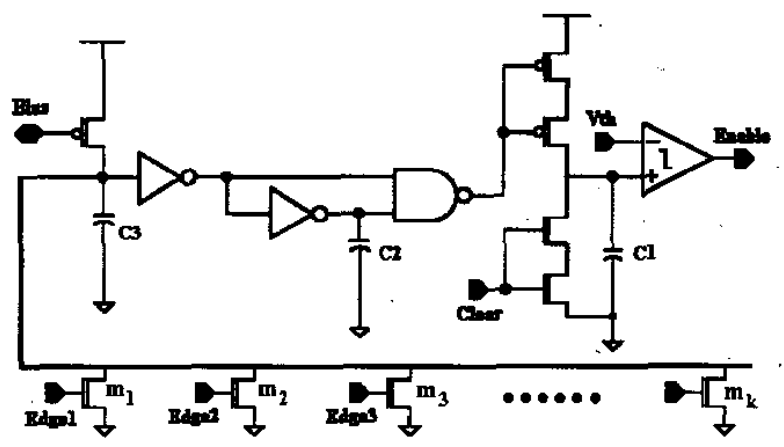

Fig. 12. Pulse fusion and decision circuit

\section{Simulation results}

Fig. 13 shows the simulation results. The first two plots of $I_{01}$ and $I_{02}$ are the light density of neighboring pixels. The time offset of the two inputs indicates movement of objects, which is detected as shown in the third plot of short 
pulses named as 'MvEdge'. All the short pulses are added together by the fusing circuit. The fused pulses are used to charge the capacitor $\mathrm{C}_{1}$, the voltage across which is shown in the fourth plot, named 'storage'. It increases step by step. After the 'storage' voltage on the capacitor reaches the threshold, $V_{t h}$, the output 'En' becomes high and trigger the digital camera, the DSP chip, and the RF driver to begin sampling and transmitting data.

The simulated average power consumption for two $16 \times 1$ motion sensor arrays is $0.506 \mu \mathrm{W}$ per cell at $100 \mathrm{pA}$ background photo current per pixel. And the pulse fusion/decision circuit cost $2.946 \mu \mathrm{W}$. Thus, the total power for the two $16 \times 1$ sensor is only $19.138 \mu \mathrm{W}$.

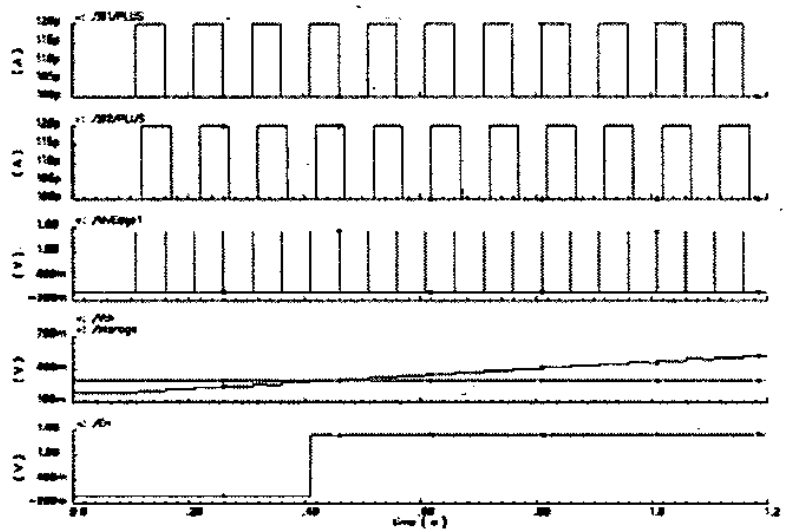

Fig. 13. Simulation results for the motion sensor

\section{SUMMARY}

This paper has presented a motion triggered ultra-low power CMOS image sensor containing two parts: digital imager and analog motion sensor. They share the same set of photosensors by an interleaved structure. Both the digital imager and the motion sensor are optimized for very low power consumption. The $64 \times 64$ pixel digital imager consumes less than $35 \mu \mathrm{W}$ at $200 \mathrm{k}$ sampling frequency, including a very low power $\mathrm{AD}$ converter which only needs $10 \mu \mathrm{W}$. The two $16 \mathrm{x} 1$ motion sensor arrays consume less than $20 \mu \mathrm{W}$ totally, which is much less than the typical work in the literature. The motion sensors act as a monitor when the system is standby and trigger the image acquisition and RF transmission when motion is detected.

\section{ACKNOWLEDGEMENT}

The work is supported by National Science Foundation project ECS-0225528.

\section{REFERENCES}

[1] Goy, J., Courtois, B., Karam, J.M., "Design of an APS CMOS image sensor for Low light level applications using standard CMOS technology", Analog Integrated Circuits and Signal Processing, Kluwer Academic Publishers, volume, 29 pages $95-104,2001$
[2] McCreary, J.L. and Gray, P.R., "All-MOS charge redistribution analog-to-digital conversion techniques I". Solid-State Circuits, IEEE Journal of, Volume, 10 Issue 6, Pages 371-379, Dec 1975

[3] Russell, H., "An improved successive approximation register design for use in A/D converters". Circuits and Systems, IEEE Transactions on, Volume. 25 Issue 7 , Pages $550-554$, Jul 1978

[4] Amaral, P., Goes, J., Paulino, N., and Steiger-Garcao, A., "An improved low-voltage low-power CMOS comparator to be used in high-speed pipeline ADCs" Circuits and Systems, 2002. ISCAS 2002. IEEE International Symposium on , Volume: 5 , 26-29, Pages V-141 -V-144 vol.5, May 2002

[5] Sumanen, L., Waltari, M., Hakkarainen, V. and Halonen, K., "CMOS Dynamic comparators for pipeline A/D converters" Circuits and Systems, 2002. ISCAS 2002. IEEE International Symposium on, Volume. 5 , 26-29, Pages V-157 -V-160 vol.5, May 2002

[6] Higgins, C.M., Deutschmann, R.A., Koch, C., "Pulsebased 2-D motion sensors". Circuits and Systems II Analog and Digital Signal Processing, IEEE Transactions on, Vol. 46, 1999 pages 677-687

[7] Ogmen, H. and Gagne, S., "Neural Network Architectures for Motion Perception and Elementary Motion Detection in the Fly Visual System," Neural Networks, vol. 3, pp. 487-505, 1990.

[8] Etienne-Cummings,R., Van der Spiegel, J., Mueller, P. and Zhang, Mao-Zhu, "A foveated silicon retina for two-dimensional tracking," IEEE Transaction on Circuits and Systems II: Analog and Digital Signal Processing, Vol. 47, Issue 6, pp. 504 -517, June 2000.

[9] Li, Z., Aizawa, K. and Hatori, M., "Implementation of a $2 \mathrm{D}$ motion vector detection on image sensor focal plane," Proceedings of the 1999 IEEE International Symposium on Circuits and Systems, Vol. 5, pp. 156 159,1999

[10] Moini, A., Bouzerdoum,A., Eshraghian, K., Yakovleff, A., Nguyen, X. T., Blanksby, A., Beare, R., Abbott, D., and Bogner, R. E., "An insect vision-based motion detection chip," IEEE Journal of Solid-State Circuit , Vol. 32, Issue: 2, pp. 279 -284, February 1997 